\title{
Statin use and risk of liver cancer: evidence from two population-based studies
}

Short title: Statin use and risk of liver cancer

Kim Tu Tran, ${ }^{1}$ Úna C. McMenamin,,${ }^{1}$ Helen G. Coleman,,${ }^{1,2}$ Chris R. Cardwell, ${ }^{1}$ Peter Murchie,${ }^{3}$ Lisa Iversen, ${ }^{3}$ Amanda J. Lee, ${ }^{4}$ Aaron P. Thrift ${ }^{5,6}$

${ }^{1}$ Cancer Epidemiology and Health Services Research Group, Centre for Public Health, Queen's University Belfast, Belfast, Northern Ireland, United Kingdom.

${ }^{2}$ Centre for Cancer Research and Cell Biology, Queen's University Belfast, Northern Ireland, United Kingdom.

${ }^{3}$ Academic Primary Care, Institute of Applied Health Sciences, University of Aberdeen, Aberdeen, United Kingdom.

${ }^{4}$ Medical Statistics Team, Institute of Applied Health Sciences, University of Aberdeen, Aberdeen, United Kingdom.

${ }^{5}$ Section of Epidemiology and Population Sciences, Department of Medicine, Baylor College of Medicine, Houston, Texas.

${ }^{6}$ Dan L Duncan Comprehensive Cancer Center, Baylor College of Medicine, Houston, Texas.

Contact Information: Aaron P. Thrift, Ph.D., Baylor College of Medicine, One Baylor Plaza, MS: BCM307, Houston, TX 77030-3498. Tel: 713-798-9107; Fax: 713-798-3658; E-mail: aaron.thrift@bcm.edu

Key words: statins, liver cancer, hepatocellular carcinoma, intrahepatic bile duct carcinoma.

Abbreviation used: HCC, hepatocellular carcinoma; IBDC, intrahepatic bile duct carcinoma; PCCIU, Primary Care Clinical Informatics Unit; ORs, odds ratios; HRs, hazard ratios; CIs, confidence intervals. 
Authorship statement: APT is the guarantor of the article. AJL, CRC, LI and PM conceived and designed the study. AJL, CRC, LI, PM and ÚCMcM were involved in data acquisition. AJL, CRC, KTT, LI, PM and ÚCMcM obtained funding for various aspects of study. CRC, KTT and ÚCMcM conducted statistical analysis. AJL, APT, CRC, HGC, KTT, LI, PM, and ÚCMcM contributed to interpretation of data. CRC acted as study supervisors. AJL, APT, CRC, HGC, KTT, LI, PM, and ÚCMcM critically revised the manuscript for important intellectual content. APT and KTT drafted the manuscript and all co-authors contributed to and agreed the final manuscript.

Conflict of interest statement: All authors have no conflict of interest to declare.

Article category: Cancer Epidemiology

Novelty and Impact: Previous studies of the inverse association of statins and liver cancer have not considered well the effect of main indication and contraindication of statins, meanwhile a study taking account of these factors found no association. Our study based on two large independent populations, different study designs, and adjusting for important covariates, found a consistent inverse association between statins and liver cancer which was only seen for hepatocellular carcinoma but not intrahepatic bile duct carcinoma. 


\section{UNSTRUCTURED SUMMARY}

Epidemiological studies of statin use and liver cancer risk have produced conflicting results. We examined the association between statin use and risk of primary liver cancer in two large independent study populations taking account of important covariates and main indications of statins such as high cholesterol and chronic liver disease. We performed a nested case-control study within the Scottish Primary Care Clinical Informatics Unit (PCCIU) database. Five controls were matched to cases with primary liver cancer and we used conditional logistic regression to calculate odds ratios (ORs) and 95\% confidence intervals (CIs) for associations with statin use. We also conducted a prospective cohort study within the UK Biobank using self-reported statin use and cancer-registry recorded primary liver cancer outcomes. Cox regression was used to calculate hazard ratios (HRs) and 95\% CIs. In the PCCIU case-control analysis, 434 liver cancer cases were matched to 2,103 controls. In the UK Biobank cohort, 182 out of 475,768 participants developed incident liver cancer. Statin use was associated with 39\% lower risk of liver cancer in the PCCIU (adjusted OR 0.61, 95\% CI 0.43-0.87). When we examined specific subtypes of liver cancer in the UK Biobank, statin use was associated with lower risk of hepatocellular carcinoma (HCC) (adjusted HR, 0.48; 95\% CI, 0.24-0.94) but not intrahepatic bile duct carcinoma (IBDC) (adjusted HR, 1.09; 95\% CI, 0.45-2.64). In conclusion, we found a consistent inverse relationship between statin use and risk of primary liver cancer which was only seen for HCC but not IBDC. 


\section{INTRODUCTION}

Liver cancer $(85-90 \%$ hepatocellular carcinoma $[\mathrm{HCC}])$ is a rapidly increasing, highly fatal cancer. ${ }^{1}$ In the United States, age-adjusted mortality rates for liver cancer increased by $43 \%$ between 2000 and 2016. ${ }^{2}$ Increasing secular trends in incidence and mortality have been observed across many countries including the United Kingdom ${ }^{3}$, Poland, Brazil, Germany and Norway. ${ }^{4}$ Major risk factors for liver cancer include hepatitis $\mathrm{C}$ virus (HCV) infection, hepatitis B virus (HBV) infection, heavy alcohol drinking, and non-alcoholic fatty liver disease (NAFLD) ${ }^{5,6}$ With further increases in liver cancer incidence predicted, ${ }^{7}$ there is growing interest in identifying modifiable risk factors for liver cancer, especially those that can be targeted with medications, such as aspirin and nonsteroidal antiinflammatory drugs (NSAIDs). Use of statins (or 3-hydroxy-3-methylglutaryl-coenzyme A reductase inhibitors), commonly used because of their efficacy in preventing cardiovascular morbidity and mortality, has been shown to be inversely related to risk of various cancers. ${ }^{8-11}$

There is increasing evidence from epidemiological studies of an inverse relationship between statin use and the risk of liver cancer. Results from recent systematic reviews and meta-analyses show that, compared with non-users of statins, individuals using statins have $40 \%-50 \%$ lower risk of liver cancer. ${ }^{11-13}$ However, there remains unexplained high between-study heterogeneity that affects the statistical validity of the summary estimates of effect reported in these studies. Stratification on, for example, histological type (HCC, intrahepatic bile duct carcinoma [IBDC]), risk factors (HCV and HBV infection, alcohol abuse, NAFLD, and type 2 diabetes), study design and study location, has provided some information on the topic. Nonetheless, other methodological considerations, risk factors, and important indications and contraindications of statin use have not been well studied.

We therefore examined the association between use of statins and the risk of primary liver cancer using data from two well-designed large, independent datasets from the UK, taking important covariates into account. 


\section{METHODS}

\section{Primary Care Clinical Informatics Unit (PCCIU) database}

\section{Data source}

The Primary Care Clinical Informatics Unit (PCCIU) database is an electronic primary care dataset that captures approximately $15 \%$ of the Scottish population from 2000 to $2011 .{ }^{14}$ Demographics and details of patient encounters, clinical diagnosis (using Read codes) ${ }^{15}$ by General Practitioners (GPs), and prescriptions were collected routinely for every patient. Data access was approved by the Research Applications and Data Management Team of the University of Aberdeen, and we obtained ethical approval for this analysis from the School of Medicine, Dentistry and Biomedical Sciences Research Ethics Committee at Queen's University Belfast (reference number: 15.43).

\section{Study design}

A nested case-control study within the PCCIU database was conducted. Cases were defined as having the first diagnosis of primary liver cancer, including HCC and IBDC, (based upon GP Read code: B15, excluding B153) between January 1, 1999 and April 30, 2011. Up to five controls were matched to each case on exact year of birth, sex and GP's practice. We defined the index date as the date of liver cancer diagnosis for cases and the date of the index case's liver cancer diagnosis for matched controls. Cases and controls had to be free from other cancer (excluding non-melanoma skin cancer) prior to their index date.

The start of prescription records was considered January 1, 1996 (as prescriptions prior to this date were less likely to have been electronically recorded) or the date of patient registration at a GP practice if this occurred after January 1, 1996. The shortest duration of available prescription records was determined within each matched set of a case and its controls. The start of the exposure period was then set as the index date minus this duration within each matched set of a case and controls to ensure all members of the matched set had an identical length of exposure period. The end of the exposure period was one year prior to the index date to reduce the potential for reverse causation due to increased exposure to healthcare professionals following cancer symptoms. ${ }^{16}$ Cases and controls 
with less than three years of prescription records prior to their index date were excluded from the current analysis.

\section{Data collection}

Medication use was determined from GP prescriptions in the exposure period. We extracted prescriptions for statins ${ }^{17}$ (including atorvastatin, fluvastatin, pravastatin, rosuvastatin, simvastatin, cerivastatin). Defined daily doses (DDD) were calculated from the quantity of tablets and strength, as defined by World Health Organization. ${ }^{18}$ Comorbidities were obtained from GP diagnosis codes prior to the index date, including diabetes, coronary heart disease, myocardial infarction, heart failure, peripheral vascular disease, cerebrovascular disease, cerebrovascular accident, mental illness, gastrooesophageal reflux disease, peptic ulcer disease, and liver diseases (hepatitis, cirrhosis, alcoholic fatty liver, non-alcoholic fatty liver, biliary cirrhosis). Proton pump inhibitors (PPIs), histamine 2-receptor antagonists (H2RA), beta-adrenoceptor blocking drugs, nonsteroidal anti-inflammatory drugs (NSAID) and aspirin use was identified from prescription records. Lifestyle risk factors were extracted from GP records including smoking status (never smoker, previous smoker, and current smoker), alcohol consumption (none, low [moderate or light drinker], or high intake [above recommended limits, chronic alcoholism]), and obesity (body mass index [BMI]>30], or not obese) using the most recent record prior to the index date. Postcode of each GP practice was used to assign deprivation fifths using the Scottish Index of Multiple Deprivation. ${ }^{19}$

\section{UK Biobank}

\section{Data source}

The UK Biobank is a cohort health resource containing approximately 500,000 volunteer participants aged 40 to 69 from England, Scotland and Wales recruited from 2006 to $2010 .{ }^{20}$ A wide range of data was collected at baseline including lifestyle, environmental, medical history and physical measures, along with biological samples. The UK Biobank is linked to cancer registry data from the Health and Social Care Information Centre (in England and Wales) and the National Health Service Central 
Register (in Scotland). The UK Biobank has ethical approval from the North West Multi-Centre Research Ethics Committee. All participants provided written informed consent.

\section{Study design}

We conducted a prospective cohort study among participants in the UK Biobank. Liver cancer cases were identified using cancer registry records (based upon ICD 10 codes C22, liver and intrahepatic bile duct cancer) up to September 30, 2014. Participants with a cancer diagnosis (apart from nonmelanoma skin cancer) prior to baseline or in the year after baseline were excluded (as these cancers may have been present at baseline). Consequently, cohort participants were followed from one year after baseline until the date of liver cancer diagnosis or censoring (on the earliest of the date of death, date of other cancer, or September 30, 2014).

\section{Data collection}

Participants reported history of statin use was collected by using a touchscreen questionnaire at baseline at UK Biobank Assessment Centres, and then verified during verbal interview with a UK Biobank nurse. Data on demographic and lifestyle factors were also determined from patient interview and touch screen at baseline, including age, sex, comorbidities (diabetes, hypertension, high cholesterol, angina, myocardial infarction, heart failure, stroke, peripheral vascular disease, cirrhosis, hepatitis, gastro-oesophageal reflux disease, peptic ulcer disease), smoking status (never smoker, previous smoker or current smoker), alcohol consumption (never, <1 day per week, 1-2 days per week, 3-4 days per week or $>4$ days per week), and other medication use (PPIs, H2RA, nitrates, NSAID, and aspirin). BMI $\left(\mathrm{kg} / \mathrm{m}^{2}\right.$ categorized as $<25,25-30$, and $\left.>30\right)$ was calculated from height and weight measurements recorded at baseline by trained research staff. The Townsend score based upon postcode of residence was determined as a measure of deprivation. ${ }^{21}$

\section{Statistical analysis}


We compared the characteristics of cases and control in PCCIU and participants who proceeded to get liver cancer and those that did not in UK Biobank using descriptive statistics (e.g., mean, median) for continuous variables and frequencies and percentages for categorical variables.

In the PCCIU dataset nested case-control study, we used conditional logistic regression models to estimate odd ratios (OR) and associated 95\% confidence intervals (CI) for the association between statin use and the risk of liver cancer. The matched design accounted for age, sex and GP practice, and we adjusted for potential confounders (e.g., smoking, obesity, alcohol consumption, co-morbidities) through a series of multivariate analyses. In addition to examining associations with ever/never use of statins, we examined associations with statin use according to the number of prescriptions, DDDs, and by type of statin therapy. Sensitivity analyses were also conducted removing prescriptions in the 2 years prior to index date (including only patients with 4 years of medical records), and in the 4 years prior to index date (including only patients with 6 years of medical records), to investigate the potential for reverse causation potentially due to gastrointestinal symptoms. A further sensitivity analysis was conducted adjusting for smoking and alcohol using multiple imputation with chained equations. ${ }^{22}$ First, an imputation model was created using ordered logit models including age, sex, statin prescribed, obesity, comorbidity, medication use, separately for cases and controls. Twenty-five imputations were conducted and results were combined using Rubin's rules. ${ }^{23}$ Another sensitivity analysis was performed by not adjusting for diabetes and aspirin use variables.

In contrast to the PCCIU database, the UK Biobank is a prospective cohort design and so the association was analyzed using Cox proportional hazards regression models. Age was the underlying time scale (individuals were considered at risk from birth and under observation from age at baseline, left truncated) and hazard ratios (HR) and associated 95\% CIs were calculated for statins use and liver cancer risk overall and by histological subtype (HCC based upon ICD 10 code C22.0 and IBDC code C22.1). In the adjusted analyses, the model contained age, sex, deprivation, BMI, alcohol, smoking, comorbidities at baseline and medication use at baseline. Sensitivity analyses were conducted by repeating the analyses starting follow-up at 2 years after baseline (to remove cancers within 2 years, 
which could have influenced medication prescribing at baseline). Another sensitivity analysis was performed by not adjusting for high cholesterol and diabetes variables.

All analyses were performed using Stata 14.0. Statistical significance was determined at $\alpha=0.05$, and all $p$-values for statistical significance were two-sided

\section{RESULTS}

\section{Primary Care Clinical Informatics Unit (PCCIU) database}

Our nested case-control study in PCCIU included 434 cases of liver cancer and 2,103 matched controls. The median exposure period was 4.8 years (interquartile range, 3.1-7.3 years) in cases and controls. Liver cancer cases were more likely than controls to smoke, consume high levels of alcohol, have diabetes, cerebrovascular disease, cerebrovascular accident, liver diseases, and use aspirin and PPIs (Table 1).

Overall, similar proportions of cases and controls used statins (25.6\% vs $27.2 \%$; Table 2$)$. In the unadjusted model, there was no significant association between statin use and risk of liver cancer (OR, 0.92; 95\% CI, 0.72-1.18) (Table 2). However, in the fully adjusted model, statin use was associated with $39 \%$ lower risk of liver cancer (fully adjusted OR, 0.61; 95\% CI, 0.43-0.87).

Further analyses revealed that differences in history of diabetes and aspirin use confounded this association and excluding both these factors from the multivariate model attenuated the inverse association with statin use (adjusted HR without aspirin and diabetes, 0.75 ; 95\% CI 0.54-1.03). The model that only included diabetes and aspirin adjustment showed a similar association with the main analysis (HR adjusted only for aspirin and diabetes, 0.66; 95\% CI 0.49-0.89). When we re-categorized cases and controls according to both history of diabetes and statin use (neither, diabetes and no statin use, no diabetes and statin use, and both diabetes and statin use), and use of aspirin and statins (neither, aspirin use and no statin use, no aspirin use and statin use, and both aspirin and statin use), 
the inverse association with statin use was only observed among those without diabetes (adjusted HR, 0.69; 95\% CI, 0.47-1.00) and those without concurrent aspirin use (adjusted HR, 0.59; 95\% CI, 0.34$1.00)$.

We found evidence for a dose-response relationship with increased duration of statin exposure (compared with never users: $1-11$ prescriptions; fully adjusted OR, $0.74 ; 95 \%$ CI, 0.46-1.17; $\geq 12$ prescriptions; OR, 0.54; 95\% CI, 0.36-0.82; $\mathrm{p}$ trend 0.003). Associations were similar when exposure was based upon DDDs. Finally, the magnitude of the inverse association with risk of liver cancer was larger for simvastatin (fully adjusted OR, $0.53 ; 95 \% \mathrm{CI}, 0.36-0.78$ ) compared to other types of statins (Table 2). In a sensitivity analysis, the association with statin use was similar to the main analysis after introducing a 2 year (fully adjusted OR, $0.63 ; 95 \%$ CI, 0.42-0.94) or 4 year (fully adjusted OR, 0.71; 95\% CI, 0.40-1.25) lag period. Associations were similar to the main analysis in other sensitivity analyses (Table 3).

\section{UK Biobank}

Among 471,851 participants in the UK Biobank, 182 participants developed incident liver cancer (88 HCC and 72 IBDC cases) over a median follow-up of 4.6 years (interquartile range, 3.9-5.3 years). Those who developed liver cancer during follow-up were more likely than those who did not develop liver cancer to be older, male, be from deprived areas, smoke, consume high level of alcohol, be obese, have diabetes, hypertension, angina, myocardial infarction, heart failure, stroke, cirrhosis, hepatitis, and use nitrates, aspirin, PPIs, and H2RA at baseline (Table 1).

Overall, amongst 395,301 statin users 137 developed liver cancer and amongst 76,550 statin nonusers 45 developed liver cancer. In the unadjusted model, there was no significant association between statin use and risk of liver cancer (HR, 1.17; 95\% CI, 0.83-1.65) (Table 4). However, similar to that observed in the PCCIU analysis, in the fully adjusted model, statin use was associated with $36 \%$ lower risk of liver cancer (fully adjusted HR, 0.64; 95\% CI, 0.39-1.07), although the association 
did not achieve statistical significance. The reduced risk was most marked for HCC (adjusted HR, 0.48; 95\% CI, 0.24-0.94) but not for IBDC (adjusted HR, 1.09; 95\% CI, 0.45-2.64).

No significant associations were observed between type of statins and risk liver cancer overall, or by histological subtype. Further analyses revealed that the reduced risk for liver cancer was largely apparent only after controlling for differences in prevalence of diabetes among cases and controls (HR adjusted only for diabetes, 0.73 ; 95\% CI 0.49-1.08). In sensitivity analyses (Table 5) introducing a 2 year lag, the association between statins and liver cancer risk (adjusted HR 0.63, 95\% CI 0.36-1.10) and HCC risk (adjusted HR 0.57, 95\% CI 0.28-1.15) were similar to the main analysis. Finally, a sensitivity analysis not adjusting for high cholesterol revealed a slightly more marked inverse association between statin use and risk of liver cancer (adjusted HR 0.52, 95\% CI 0.33-0.80) and risk of HCC (adjusted HR 0.36, 95\% CI 0.19-0.67). 


\section{DISCUSSION}

Using data from two large independent population-based studies, we found a consistent inverse relationship between use of statins and the risk of primary liver cancer, although this was only evident for HCC and not IBDC in the UK Biobank analysis. Compared with statin non-users, individuals with a history of any statin use had an over $35 \%$ lower risk of developing incident liver cancer. Importantly, our findings are evident in both study designs (we observed similar results in casecontrol vs. prospective cohort study) and method of exposure ascertainment (we observed similar results for self-reported data vs. prescription records).

Our results from the PCCIU database nested case-control and UK biobank (particularly for HCC) studies are consistent with previous studies. A reduction of liver cancer risk in statin users has been observed in meta-analyses ${ }^{11,24,25}$ with the most recent ${ }^{11}$ observing a $54 \%$ reduction in liver cancer risk and a reduction of $14 \%$ in liver cancer risk per 50 daily defined doses. Our study result from PCCIU database is similar to a previous study with the same study design in a UK population, that observed a reduced liver cancer risk in statins users and similarly, the association followed a dose response. ${ }^{26}$ However, our results are an important addition to the literature as this previous UK-based study, unlike ours, was unable to verify cancer outcomes through cancer registries and was unable to examine associations with specific histological subtypes of liver cancer.

In UK Biobank, the inverse association was less apparent after adjustment for cholesterol, which has been shown to reduce liver cancer risk, ${ }^{27}$ but very few previous studies have adjusted for cholesterol..$^{28}$ For IBDC, our study showed no significant association with use of statins. This is not consistent with the literature. Two previous case-control studies found consistently reduced risk for bile duct carcinoma (adjusted OR 0.87, 95\% CI 0.75-1.01) ${ }^{10}$ and cholangiocarcinoma (adjusted OR 0.80, 95\% CI 0.71-0.90). ${ }^{29}$ The difference in the result between our study and two previous studies could be explained partly by the limited number of IBDC cases in the UK Biobank database. Moreover, the two previous studies investigated cholangiocarcinoma defined as either extra- or intrahepatic bile duct carcinoma, while in our study we investigated specifically IBDC. For statin subtypes, only 
simvastatin was significantly associated with reduced liver cancer risk in PCCIU dataset which might be explained by its frequency of use in comparison to other statin types. Although the HR was reduced, we did not find a significant association for simvastatin use in UK Biobank. Simvastatin was found to be inversely associated with liver cancer in a previous meta-analysis, however, similar associations were observed for other statin subtypes. ${ }^{24}$

The cause of the observed reduced risk of liver cancer in statin users is unknown. Nevertheless, some potential mechanisms for a protective effect of statins on cancer can be suggested. Statins have been shown to inhibit the production of mevalonate leading to a reduction in the growth and proliferation of neoplastic cells. ${ }^{30}$ Statins have also been shown to inhibit inflammation ${ }^{30}$ and angiogenesis, ${ }^{31}$ which play a crucial role in carcinogenesis. Lastly, statins have been shown to induce apoptosis of abnormal cells by regulating the signaling pathways including the RAF-MEK-ERK pathway. ${ }^{30} \mathrm{~A}$ more specific look at mechanism of statins on liver cancer showed that statins might involve the ubiquinone inhibition, leading to the apoptosis of preneoplastic liver cells. ${ }^{32}$ Alternatively, the inverse associations could reflect confounding. High cholesterol is the main indication for statin treatment and has been shown to be inversely associated with risk of liver cancer. ${ }^{27,33}$ In UK Biobank we adjusted for high cholesterol and the associations with HCC remained, but high cholesterol was based upon self-report and we did not have actual cholesterol serum levels and therefore there remains the potential for residual confounding by cholesterol. Furthermore, although statins appear safe in patients with chronic liver disease,$^{34}$ it is possible that patients with liver diseases may be less likely to be prescribed statins because of their potential hepatotoxicity. ${ }^{35}$ Our study adjusted for chronic liver diseases but we did not have information on liver function tests, which are a known contraindication for statins,${ }^{36}$ therefore we cannot rule out confounding by liver function test results, as has been seen previously. ${ }^{28}$

Our study has a number of strengths. First, in PCCIU database, information on the number of prescriptions, medication dose, and number of pills were available so we could investigate any potential dose response relationship between statins and liver cancer. Recall bias was limited because 
medication use was recorded by GPs. Second, in the UK Biobank, cases were identified by cancer registry therefore information of outcome was verified. Details of histological type of liver cancer were also provided allowing us to demonstrate that statins were only associated with a reduced incidence of HCC. While the medication use was based on self-report, the information was verified by a trained nurse. More importantly, in both datasets we have adjusted for a wide range of covariates including the main risk factors for liver cancer (age, sex, BMI, smoking and alcohol consumption, comorbidities), indications and contraindications of statins (high cholesterol, chronic liver diseases), and medications previously shown to be associated with liver cancer risk (metformin ${ }^{37,38}$, aspirin ${ }^{39}$, beta-blockers ${ }^{40}$, proton pump inhibitors and histamine-2 receptor antagonist ${ }^{41}$ ).

However, our study has a number of limitations. We cannot rule out confounding by incomplete or unavailable confounders e.g. cholesterol and liver function tests as previously mentioned. Therefore, further studies should aim to collect detailed information on these variables. Furthermore, we cannot be sure that participants used the prescribed medications and previous studies have shown evidence of non-adherence to statin therapy regimes because of a lack of knowledge about their efficacy and concerns about side effects. ${ }^{42}$ However this information bias seems more likely to drag associations to the null rather than to produce the inverse associations we observed. Moreover, as data on histological type of liver cancer was not available in PCCIU database we were unable to examine separately the association of statin use with risk of HCC and IBDC. A further potential limitation in the PCCIU study is that we adjusted for the most recent recorded lifestyle risk factors prior to cancer diagnosis, which could lead to inappropriate adjustment if these lifestyle risk factors changed due to a predisposing condition such as cirrhosis.

In conclusion, results from our two independent datasets showed a decreased risk of liver cancer associated with statin use, which was limited to the HCC subtype in one study. However, whether this inverse association reflects a true causal relationship remains unclear and requires further study. 
Acknowledgements: The analysis of UK Biobank has been conducted using the UK Biobank Resource under Application Number 34374. We acknowledge collaboration with the Research Applications and Data Management Team lead by Ms Katie Wilde, University of Aberdeen in conducting this study. KTT is supported by the Vietnam International Education Cooperation Department. Access to PCCIU data was provided by Queen's University Belfast and the Centre for Academic Primary Care, University of Aberdeen. Access to the UK Biobank was funded by a Cancer Research UK Population Research Postdoctoral Fellowship awarded to ÚCMcM.

\section{REFERENCES}

1. White DL, Thrift AP, Kanwal F, et al. Incidence of Hepatocellular Carcinoma in All 50 United States, From 2000 Through 2012. Gastroenterology. 2017;152(4):812-820.

2. Xu J. Trends in Liver Cancer Mortality Among Adults Aged 25 and Over in the United States, 2000-2016. NCHS Data Brief, no 314 Hyattsville, MD: National Center for Health Statistics. 2018.

3. Ladep NG, Khan SA, Crossey MM, et al. Incidence and mortality of primary liver cancer in England and Wales: Changing patterns and ethnic variations. World J Gastroenterol. 2014;20(6):1544-1553.

4. Martin C. S.Wong, Johnny Y. Jiang, William B Goggins, et al. International incidence and mortality trends of liver cancer: a global profile. Scientific Reports. 2017;7:45846.

5. Mittal S, Sada YH, El-Serag HB, et al. Temporal Trends of Nonalcoholic Fatty Liver DiseaseRelated Hepatocellular Carcinoma in the Veteran Affairs Population. Clin Gastroenterol Hepatol. 2015;13(3):594-601.e1.

6. Kanwal F, R. Kramer J, Mapakshi S, et al. Risk of Hepatocellular Cancer in Patients With Non-Alcoholic Fatty Liver Disease. Gastroenterology. 2018.

7. Smittenaar CR, Petersen KA, Stewart K, et al. Cancer incidence and mortality projections in the UK until 2035. Br J Cancer. 2016;115(9):1147-1155.

8. Archibugi L, Giorgio Arcidiacono P, Capurso G. Statin use is associated to a reduced risk of pancreatic cancer: A meta-analysis. Dig Liver Dis. 2018.

9. Langballe R, Deirdre Cronin-Fenton, Dehlendorff C, et al. Statin use and risk of contralateral 
breast cancer: a nationwide cohort study. British Journal of Cancer. 2018;119:1297-1305.

10. Liu Z, Alsaggaf R, McGlynn KA, et al. Statin use and reduced risk of biliary tract cancers in the UK Clinical Practice Research Datalink. Gut. 2018.

11. Yi C, Song Z, Wan M, et al. Statins intake and risk of liver cancer: A dose-response meta analysis of prospective cohort studies. Medicine (Baltimore). 2017;96(27):e7435.

12. Singh S, Paul Singh P, Goyal Singh A, et al. Statins are associated with a reduced risk of hepatocellular cancer: a systematic review and meta-analysis. Gastroenterology. 2013;144(2):323-332.

13. Pradelli D, Soranna D, Scotti L, et al. Statins and primary liver cancer: a meta-analysis of observational studies. European Journal of Cancer Prevention. 2013;22(3):229-234.

14. University of Aberdeen The Institute of Applied Health Sciences. Primary Care Clinical Informatics Unit Research. https://www.abdn.ac.uk/iahs/research/primarycare/pcciur/index.php. Accessed May 3, 2018.

15. Scottish Clinical Information Management in Practice. SCIMP Guide to Read Codes, Read Version 2, 5 byte. https://www.scimp.scot.nhs.uk/better-information/clinical-coding/scimpguide-to-read-codes. Accessed January 17, 2019.

16. Pottegård A, Hallas J. New use of prescription drugs prior to a cancer diagnosis. Pharmacoepidemiol Drug Saf. 2017;26(2):223-227.

17. British Medical Association, Royal Pharmaceutical Society. British National Formulary. 60th ed.; 2010.

18. World Health Organisation. World Health Organisation Collaborating Centre for Drug Statistics and Methodology. http://www.who.int/medicines/regulation/medicinessafety/toolkit_ddd/en/. Accessed May 12, 2018.

19. Donnelly R. Scottish Index of Multiple Deprivation 2009: General Report. Edinburgh, UK: Office of the Chief Statistician (The Scottish Government) 2009.

20. UK Biobank. About UK Biobank. http://www.ukbiobank.ac.uk/about-biobank-uk/. Accessed February 2, 2018.

21. Whitelegg J. Health and Deprivation: Inequality and the North. Sociology of Health \& Illness. 
1988;10(3):314-315. doi:10.1111/1467-9566.ep11340224

22. White IR, Royston P, Wood AM. Multiple imputation using chained equations: Issues and guidance for practice. Statistics in Medicine. 2011;30(4):377-399. doi:10.1002/sim.4067

23. Sterne JAC, White IR, Carlin JB, et al. Multiple imputation for missing data in epidemiological and clinical research: potential and pitfalls. BMJ (Clinical research ed). 2009;338:157-160. doi:10.1136/bmj.b2393

24. Zhong G-C, Liu Y, Ye Y-Y, et al. Meta-analysis of studies using statins as a reducer for primary liver cancer risk. Sci Rep. 2016;6:26256.

25. Zhou Y-Y, Zhu G-Q, Wang Y, et al. Systematic review with network meta-analysis: statins and risk of hepatocellular carcinoma. Oncotarget. 2016;7:21753-21762.

26. McGlynn KA, Hagberg K, Chen J, et al. Statin Use and Risk of Primary Liver Cancer in the Clinical Practice Research Datalink. J Natl Cancer Inst. 2015;107(4):djv009.

27. Borena W, Strohmaier S, Lukanova A, et al. Metabolic risk factors and primary liver cancer in a prospective study of 578,700 adults. International Journal of Cancer. 2012;131(1):193-200.

28. Friedman GD, Achacoso N, Fireman B, et al. Statins and Reduced Risk of Liver Cancer: Evidence for Confounding. Journal of the National Cancer Institute. 2016;108(10):djw109.

29. Peng Y-C, Lin C-L, Hsu W-Y, et al. Statins are associated with a reduced risk of cholangiocarcinoma: a population-based case-control study. Br J Clin Pharmacol. 2015;80(4):755-761.

30. Demierre M-F, Higgins PDR, Gruber SB, et al. Statins and cancer prevention. Nat Rev Cancer. 2005;5(12):930-942.

31. Weis M, Heeschen C, Glassford AJ, et al. Statins have biphasic effects on angiogenesis. Circulation. 2002;105:739-745.

32. Börkhem-Bergman L, Acimovic J, Torndal U-B, et al. Lovastatin Prevents Carcinogenesis in a Rat Model for Liver Cancer. Effects of Ubiquinone Supplementation. Anticancer Research. 2010;30(4):1105-1112.

33. Kitahara CM, González AB de, Freedman ND, et al. Total Cholesterol and Cancer Risk in a Large Prospective Study in Korea. J Clin Oncol. 2011;29(12):1592-1598. 
34. Chang F, Wang Y, Lang H, et al. Statins decrease the risk of decompensation in hepatitis B virus- and hepatitis C virus-related cirrhosis: A population-based study. Hepatology. 2017;66(3):896-907.

35. Russo MW, Hoofnagle JH, Gu J, et al. Spectrum of statin hepatotoxicity: Experience of the drug-induced liver injury network. Hepatology. 2014;60(2):679-686.

36. The Food and Drug Administration. LIPITOR ${ }^{\circledR}$ (atorvastatin calcium). https://www.accessdata.fda.gov/drugsatfda_docs/label/2009/020702s056lbl.pdf. Accessed December 12, 2018.

37. Zhang Z-J, Zheng Z-J, Shi R, et al. Metformin for liver cancer prevention in patients with type 2 diabetes: a systematic review and meta-analysis. The Journal of Clinical Endocrinology \& Metabolism. 2012;97(7):2347-2353.

38. Murff HJ, Roumie CL, Greevy RA, et al. Metformin use and incidence cancer risk: evidence for a selective protective effect against liver cancer. Cancer Causes \& Control. 2018;29(9):823-832.

39. Simon TG, Ma Y, Ludvigsson JF, et al. Association Between Aspirin Use and Risk of Hepatocellular Carcinoma. JAMA Oncol. 2018;4(12):1683-1690.

40. Patel H, Abhishek K, Shah N, et al. Role of Non-Selective Beta Blockers in Hepatocellular Carcinoma: An Analysis in Patients with Cirrhosis and Portal Hypertension. North American Journal of Medicine and Science. 2015;8:105-108.

41. Tran KT, McMenamin ÚC, Hicks B, et al. Proton pump inhibitor and histamine-2 receptor antagonist use and risk of liver cancer in two population-based studies. Aliment Pharmacol Ther. 2018;48(1):55-64.

42. Wouters H, Dijk L Van, Geers HCJ, et al. Understanding Statin Non-Adherence: Knowing Which Perceptions and Experiences Matter to Different Patients. PLoS One. 2016;11(1):e0146272. 